\title{
Elaboration and Characterization of Composite Materials Based on Plaster-Gypsum and Mineral Additives for Energy Efficiency in Buildings ${ }^{\dagger}$
}

\author{
Said Bouzit * and Mohamed Taha \\ Faculty of Science, Ibn Zohr University, 80000 Agadir, Morocco; taha1966@hotmail.com \\ * Correspondence: saidbouzit19@gmail.com \\ + Presented at the 1st International Conference on Smart Materials for Sustainable Construction-SMASCO \\ 2019, Luleå, Sweden, 10-12 December 2019.
}

Published: 18 November 2019

\begin{abstract}
The building sector is one of the largest energy consumers in the world, prompting scientific researchers to find solutions to the problem. The choice of appropriate building materials presents a considerable challenge for improving the thermal comfort of buildings. In this scenario, plaster-based insulating materials have more and more interests and new applications, such as insulating coatings developing the building envelope. Several works are being done to improve energy efficiency in the building sector through the study of building materials with insulation quality and energy savings. In this work, new composite materials, plaster-gypsum with mineral additives are produced and evaluated experimentally to obtain low-cost materials with improved thermo-physical and acoustic properties. The resulting composites are intended for use in building walls. Plaster-gypsum is presented as a high-performance thermal material, and mineral additives are of great importance because of their nature and are environmentally friendly. Measurements of thermal properties are carried and measurements of acoustic properties. The results show that it is possible to improve the thermal and acoustic performance of building material by using plaster as a base material and by incorporating thermal insulators. The thermal conductivity of plaster alone is greater than that of plaster with mineral additives, offer interesting thermal and acoustic performance. By varying the additives, the thermal conductivity changes. Finally, comparing the results, plaster with mineral additives is considered the best building material in this study
\end{abstract}

Keywords: moroccan plaster-gypsum; mineral additives; building material; thermal conductivity; acoustic performance 